\title{
Radiation recall dermatitis triggered by sorafenib after radiation therapy for hepatocellular carcinoma
}

\author{
Gwi Eon Kim, MD, PhD', Hee-Sung Song, MD², Ki Jung Ahn, MD, PhD' , Young Suk Kim, MD \\ Departments of ${ }^{1}$ Radiation Oncology and ${ }^{2}$ Nuclear Medicine, \\ Jeju National University Hospital, Jeju National University School of Medicine, Jeju; \\ ${ }^{3}$ Department of Radiation Oncology, Inje University Busan Paik Hospital, Inje University of Medicine, Busan, Korea
}

\begin{abstract}
Sorafenib is widely used for unresectable and metastatic hepatocellular carcinomas. Radiation recall dermatitis (RRD) is an acute inflammatory reaction confined to previously irradiated skin that occurs after the administration of certain drugs. RRD after sorafenib treatment is rare; five cases have been reported thus far. We describe a 44-year-old man irradiated for chest wall bone metastasis from hepatocellular carcinoma. Eight days after radiotherapy completion, systemic therapy for metastatic hepatocellular carcinoma was initiated with sorafenib treatment. Eleven days after starting sorafenib, the patient complained of erythematous rash with pruritus in the chest wall, in a location consistent with the previous radiation field. Sorafenib was continued at the same dose, despite the RRD. The skin reaction subsided over the next 2 weeks without any medical intervention.
\end{abstract}

Keywords: Sorafenib, Radiotherapy, Radiation recall dermatitis, Hepatocellular carcinoma

\section{Introduction}

Radiation recall dermatitis (RRD) is an acute inflammatory reaction confined to previously irradiated skin, which occurs after the administration of certain drugs, mainly chemotherapeutics. The reaction presents days or even years after radiotherapy [1-4].

Sorafenib is a multi-kinase inhibitor that induces tumor cell apoptosis in hepatocellular carcinoma [5]. Adverse skin reactions, including rash, dryness, and hand-foot skin reaction, are frequently reported with sorafenib use [6]. Here, we present a rare case of sorafenib-induced RRD in a patient irradiated for bone metastasis from hepatocellular carcinoma.

\section{Case Report}

In January 2014, a 44-year-old male Asian (weight, $61 \mathrm{~kg}$; height, $168 \mathrm{~cm}$; body mass index, $21.6 \mathrm{~kg} / \mathrm{m}^{2}$ ) with chronic hepatitis B had lost $10 \mathrm{~kg}$ in the preceding 2 months. His serum alpha-fetoprotein level was $>20,000.0 \mathrm{ng} / \mathrm{mL}$. Dynamic computed tomography of the liver showed multiple arterial enhancing masses with delayed washout in the right hepatic lobe. 18F-fluoro-2-deoxy-D-glucose positron emission tomography showed hypermetabolic lesions in the right liver, right seventh anterior rib, and right iliac bone. Upon diagnosis of stage IV hepatocellular carcinoma, a transcatheter arterial chemoembolization was performed for the multiple intrahepatic metastases. After chemoembolization,

Received 22 June 2017, Revised 14 August 2017, Accepted 16 August 2017.

Correspondence: Young Suk Kim, MD, Department of Radiation Oncology, Jeju National University Hospital, Jeju National University School of Medicine, 15 Aran 13-gil, Jeju 63241, Korea. Tel: +82-64-717-1330, Fax: +82-64-717-1169, E-mail: yskim@ jejunuh.co.kr

(c) This is an Open Access article distributed under the terms of the Creative Commons Attribution Non-Commercial License (http://creativecommons.org/ licenses/by-nc/4.0/) which permits unrestricted non-commercial use, distribution, and reproduction in any medium, provided the original work is properly cited.

www.e-roj.org 

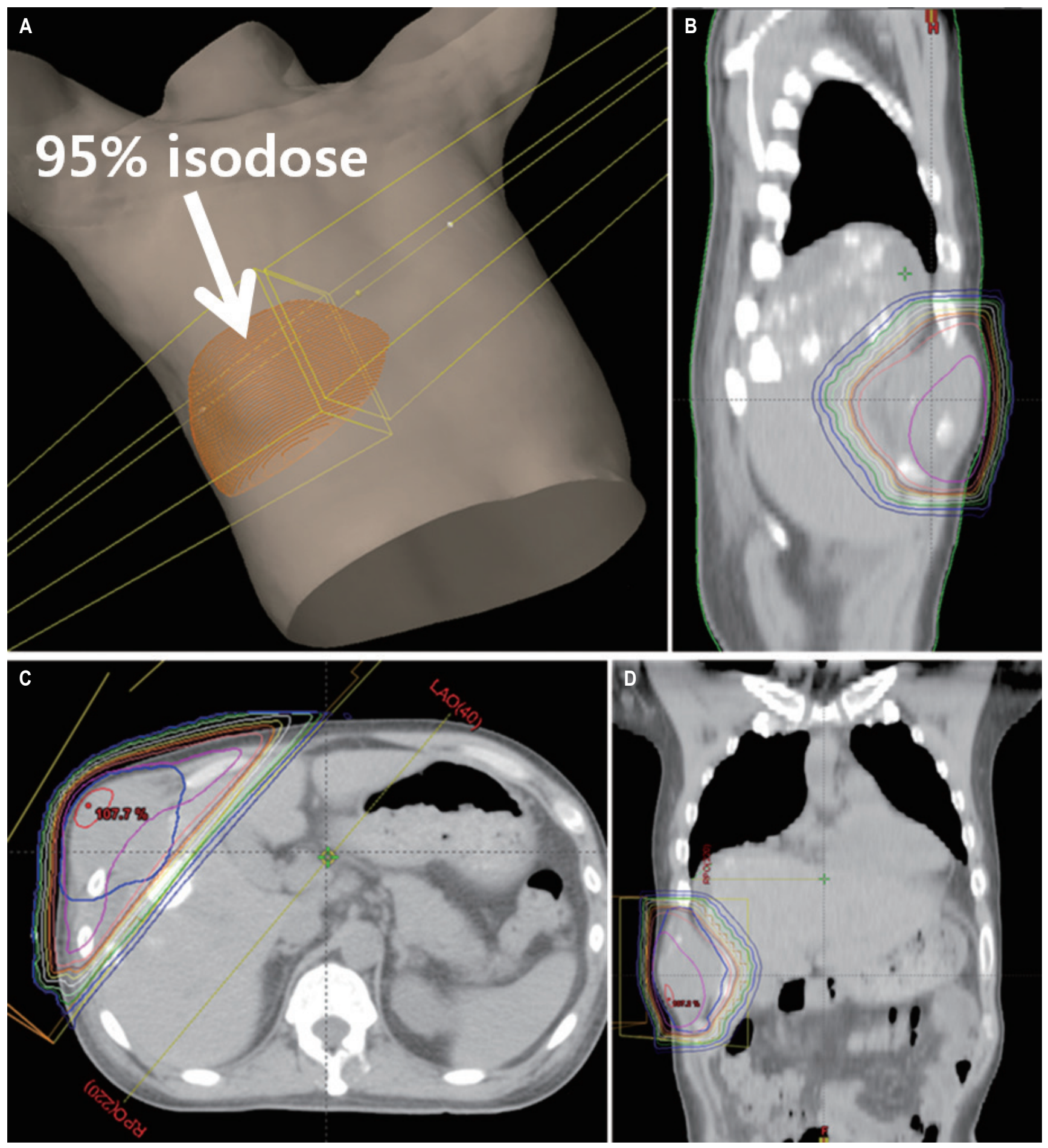

Fig. 1. Radiotherapy planning with a parallel opposing field. (A) Three-dimensional model view, (B) sagittal view, (C) axial view, and (D) coronal view.

the patient received palliative radiotherapy at a dose of 30 Gy in 10 fractions to the right seventh rib mass due to severe bone pain. Parallel opposing fields with wedge filters were used (Fig. 1). There was no skin reaction on the final day of radiation therapy. Eight days after radiotherapy completion, systemic therapy for metastatic hepatocellular carcinoma began with sorafenib at $400 \mathrm{mg}$ taken orally twice daily. Longterm comedication included tenofovir for chronic hepatitis B. 

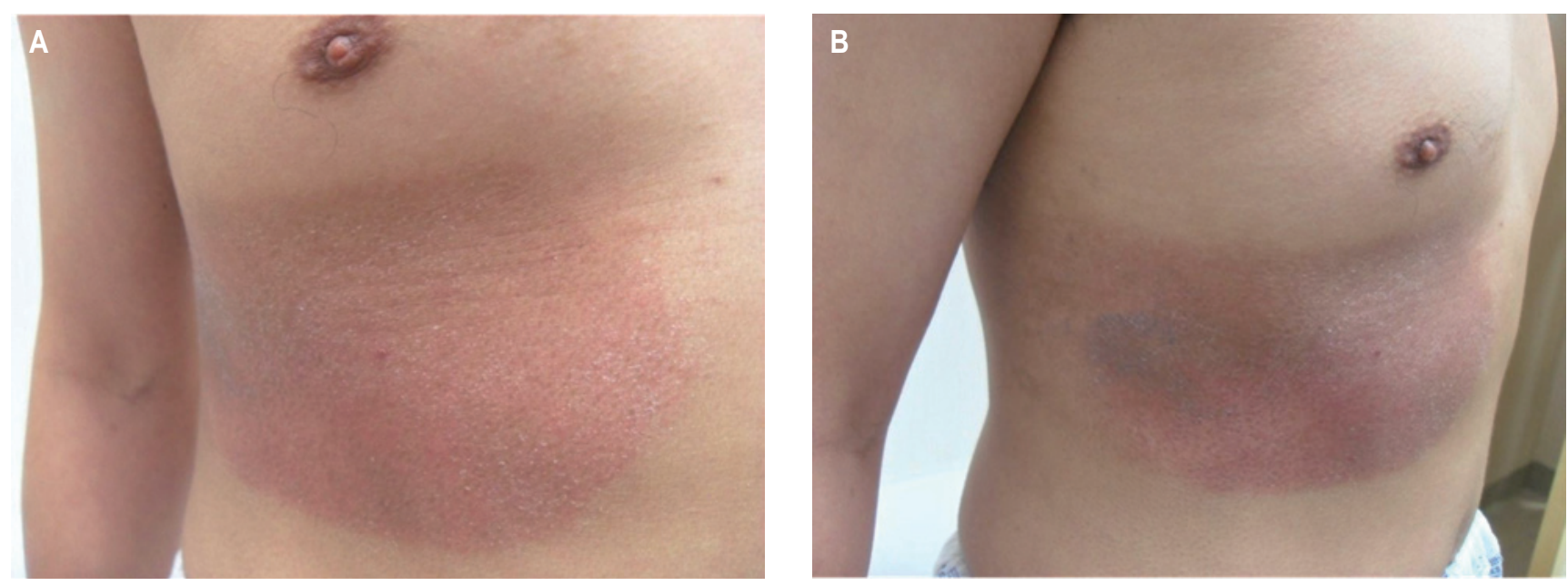

Fig. 2. Correlation of radiation recall dermatitis (right seventh rib area) with radiation fields. $(A, B)$ Photography was performed 20 days after initiation of sorafenib (28 days after radiotherapy).

Several days after starting sorafenib, the patient developed diarrhea, erythematous swelling of finger tips and greyish blisters on the palms of his hands grade 2 according to the Common Terminology Criteria for Adverse Events (CTCAE) version 4.03. These adverse events subsided spontaneously over the next 2 weeks. Eleven days after starting sorafenib (19 days after radiotherapy), the patient complained of erythematous patch with pruritus (dermatitis CTCAE grade 1) in the right seventh anterior rib area. The patient was only taking sorafenib and tenofovir at this time. There were no obvious signs of infection (fever, pain, swelling, or warmth). White blood cell count was normal $(10,000 / \mu \mathrm{L})$. C-reactive protein level was mildly elevated $(2.62 \mathrm{mg} / \mathrm{dL}$; normal range, 0.00 to $0.30 \mathrm{mg} / \mathrm{dL}$ ). When assessed by radiation oncology 20 days after starting sorafenib (28 days after radiotherapy), there was patchy erythematous hyperpigmentation and dry desquamation (dermatitis CTCAE grade 1) in the area, consistent with the patient's earlier radiation treatment fields, consistent with RRD (Fig. 2). RRD developed in the 95\% isodose area. Erythematous patch with pruritus in the area was improving at that time. Sorafenib treatment continued at the same dose of $400 \mathrm{mg}$ twice daily, despite the RRD. The skin reaction subsided over the next 2 weeks without any medical intervention.

\section{Discussion and Conclusion}

RRD is an inflammatory skin reaction within previously irradiated volume after administration of certain promoting agents, such as antineoplastic drugs [5]. Sorafenib is a small- molecule Raf kinase and vascular endothelial growth factor receptor kinase (VEGFR-2 and VEGFR-3) inhibitor. In addition, sorafenib demonstrated significant activity against several receptor tyrosine kinases involved in neovascularization and tumor progression, including platelet-derived growth factor receptor $\beta$, Flt-3, and c-KIT [7]. Sorafenib is widely used for unresectable and metastatic hepatocellular carcinomas since 2007. To our knowledge, only five cases of RRD associated with sorafenib have been reported [2-6] (Table 1). This case report highlights the clinical presentation, time course, and management of RRD in one patient receiving sorafenib after radiotherapy for bone metastasis from hepatocellular carcinoma.

The pathogenesis of RRD remains unclear. Bostrom et al. [8] have suggested that local vascular permeability or proliferative change induced by the radiotherapy may affect the subsequent pharmacokinetics of certain drugs to elicit RRD. Phillips and Fu [9] suggested that the radiation may change inherent sensitivity to the drug through various mechanisms, such as an alteration in the cell age distribution. Oanta and Irimie [10] suggested that radiotherapy induces the release of certain cytokines, including interleukin-1, interleukin-6, platelet-derived growth factor-beta, tumor necrosis factor, and transforming growth factor, causing an inflammatory response. After irradiation, cells continue to secrete low levels of cytokines; administration of certain medications causes these cytokines to increase their activity, resulting in skin reaction [10]. Camidge and Price [1] proposed that the rarity, speed of onset and extreme drug specificity of RRD support a mechanism of action based not on the triggering 


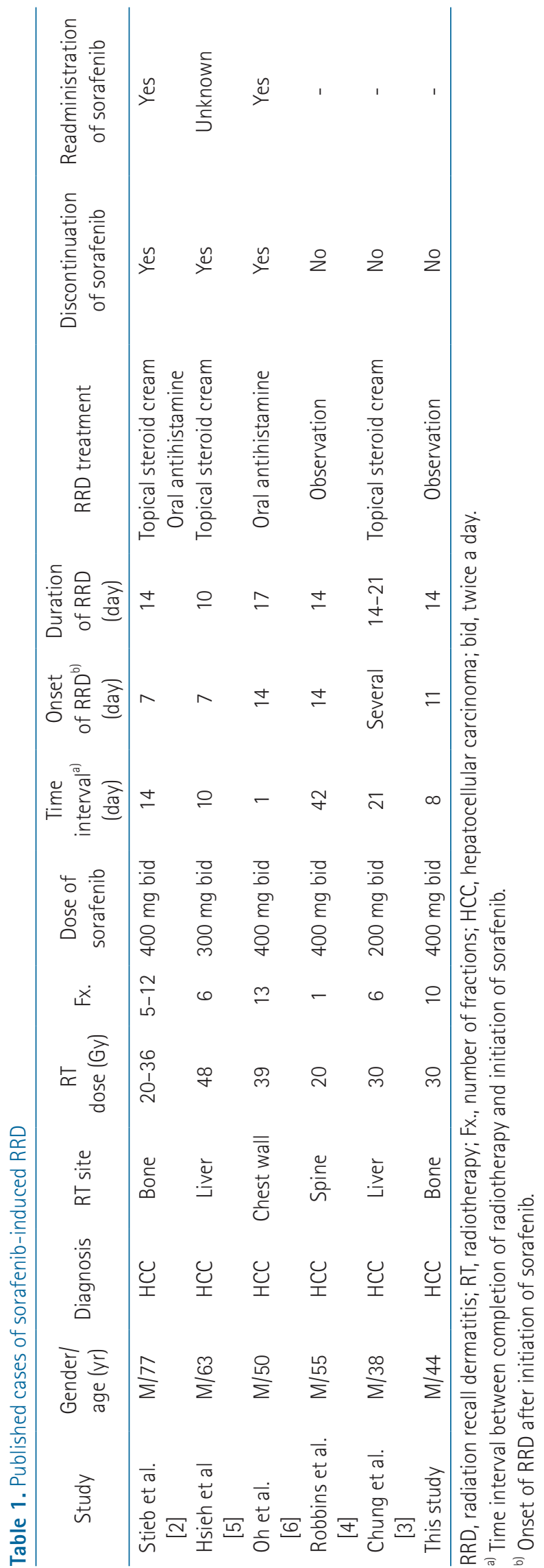

drug's cytotoxicity, but on idiosyncratic drug hypersensitivity reactions instead. Radiation may induce transient tumor and normal tissue hypoxia, stimulate VEGF production and VEGFR-2 expression, and promote angiogenesis [11]. Use of antiangiogenic agent (sorafenib) after radiotherapy may impair this angiogenesis leading to erythema and desquamation in the irradiated fields [4].

Histological findings of sorafenib-induced RRD were reported in only one case [2]. Skin biopsy of an erythematous lesion revealed diffuse perivascular lymphohistiocytic infiltrate with few eosinophils in the corium, and apoptotic keratinocytes and distinct vacuolization in the junction zone [2]. The histological picture mimicked the pattern of graftversus-host reaction of the skin or cutaneous drug allergies [2]. The pathologic finding of sorafenib-induced skin reaction shows varying degree of keratinocyte damage, while that of acute radiation dermatitis shows an inflammatory reaction with wound healing process $[6,12,13]$. Interestingly, radiation recall dermatitis can have both pathological features observed in skin reactions from the targeted therapies with dyskeratotic keratinocytes and an inflammatory component with mononuclear cells and neutrophils, as observed in acute radiation dermatitis [3].

In the prior reports, the patients were diagnosed with sorafenib-induced RRD based on the following clinical findings: (1) localized symptoms of an acute inflammatory skin reaction CTCAE grade 1-3 (including painless erythematous lesion, dry desquamation, pruritus, and patchy hyperpigmentation) within previously irradiated volume, (2) skin reaction development during sorafenib treatment, (3) no other medication provoking $R R D$, and (4) negative test results for local skin infection [2-6].

The patient in our study also experienced localized acute inflammatory symptoms in the irradiated area without evidence of infection during sorafenib treatment. The patient had been taking tenofovir (antiviral agent) after diagnosis of hepatocellular carcinoma. He received radiotherapy at 50 days after beginning of tenofovir. He didn't have any medications except for sorafenib and tenofovir before the RRD. Because tenofovir started before radiotherapy and continued during radiotherapy without any skin reactions, tenofovir is less likely to cause RRD. In the literature review, there is no evidence of RRD related with tenofovir. We did not perform the skin biopsy, but it may be helpful [6].

Previous studies reported that RRD occurred several days to 2 weeks after sorafenib administration [2-6]. In three of five reported cases, the symptoms improved in about 2 weeks after stopping sorafenib administration $[2,5,6]$. Two patients 
continued sorafenib without dose reduction during $\operatorname{RRD}[3,4]$. The pruritus and skin changes resolved over 2 to 3 weeks (Table 1).

Skin is the major site of radiation recall phenomenon [14]. Rarely, radiation recall phenomena have been described in different internal organs, including the lungs, esophagus, central nervous system (brainstem and optic nerve), gastrointestinal tract, musculoskeletal systems, heart, bladder, salivary glands, supraglottis, vagina, and oral mucosa [1417]. Ge et al. [18] emphasized that RRD may be the most visible sign of radiation recall affecting internal organs. In five cases of RRD in thoracic malignancies, RRD was accompanied by clinical and/or radiographic evidence of internal organ involvement [18].

In general, it is considered to be difficult to distinguish between a radiation-induced skin disorder and RRD. Nonetheless, in clinical practice, the two conditions are often differentiated by focusing on the specific clinical course of RRD and on the fact that the rash appears following the administration of certain drugs [19].

As the gap between radiotherapy and exposure to the recalltriggering drug narrows, inevitably there is a potential overlap of RRD with radiosensitization (involving enhancement of the initial radiation effect) [1]. Camidge and Price [1] suggested that skin reactions brought about by drugs given less than 7 days after radiotherapy should be considered radiosensitization rather than radiation recall. Oh et al. [6] reported that sorafenib was started on the day following completion of radiotherapy and a skin rash matched to the irradiated field developed 14 days after starting sorafenib (Table 1). He suggested that this skin reaction may be associated with RRD as well as acute radiation dermatitis enhanced by sorafenib (radiosensitization) because of the short time interval between radiotherapy and exposure to sorafenib in his case [6]. In other four cases, the interval between completion of radiotherapy and exposure to sorafenib was 10 to 42 days [2-5]. In our case, the interval between completion of radiotherapy and exposure to sorafenib was 8 days. There is a potential overlap of RRD with radiosensitization.

Camidge and Price [1] also proposed that an additional element in the definition of RRD would be that all acute radiotherapy reactions in the skin have completely recovered before any drug exposure triggering RRD. In our case, there was no skin reaction on the final day of radiation therapy.

Treatments for sorafenib-induced RRD include topical steroid cream, oral antihistamine, and discontinuation of sorafenib. Re-administration of sorafenib at a reduced dosage or with oral antihistamine does not lead to recurrence of RRD $[2,6]$. In our case, we did not apply topical steroid cream or oral antihistamine and continued sorafenib administration because the skin reaction was deemed tolerable [4]. Chung et al. [3] also continued the use of sorafenib with topical steroid cream treatment. Sorafenib continuation or rechallenge after discontinuation could be considered, depending on the severity of RRD in a given case.

In conclusion, it seems that RRD incidence is very low with sorafenib use. However, clinicians should be aware of the possibility of sorafenib-induced RRD to provide an appropriate treatment.

\section{Conflict of Interest}

No potential conflict of interest relevant to this article was reported.

\section{Acknowledgments}

This research was supported by the 2017 scientific promotion program funded by Jeju National University.

\section{References}

1. Camidge $R$, Price $A$. Characterizing the phenomenon of radiation recall dermatitis. Radiother Oncol 2001;59:237-45.

2. Stieb S, Riesterer 0 , Brussow C, Pestalozzi B, Guckenberger $M$, Weiler $S$. Radiation recall dermatitis induced by sorafenib : A case study and review of the literature. Strahlenther Onkol 2016;192:342-8.

3. Chung C, Dawson LA, Joshua AM, Brade AM. Radiation recall dermatitis triggered by multi-targeted tyrosine kinase inhibitors: sunitinib and sorafenib. Anticancer Drugs 2010;21:206-9.

4. Robbins J, Wollner I, Ryu S. Sorafenib induced radiation recall dermatitis after spine radiosurgery. J Radiosurg SBRT 2011;1:71-4.

5. Hsieh CH, Lin SC, Shueng PW, Kuo DY. Recall radiation dermatitis by sorafenib following stereotactic body radiation therapy. Onco Targets Ther 2014;7:1111-4.

6. Oh D, Park HC, Lim HY, Yoo BC. Sorafenib-triggered radiation recall dermatitis with a disseminated exanthematous reaction. Radiat Oncol J 2013;31:171-4.

7. Wilhelm SM, Carter C, Tang L, et al. BAY 43-9006 exhibits broad spectrum oral antitumor activity and targets the RAF/MEK/ERK pathway and receptor tyrosine kinases 
involved in tumor progression and angiogenesis. Cancer Res 2004;64:7099-109.

8. Bostrom A, Sjolin-Forsberg G, Wilking N, Bergh J. Radiation recall: another call with tamoxifen. Acta Oncol 1999;38:955-9.

9. Phillips TL, Fu KK. Quantification of combined radiation therapy and chemotherapy effects on critical normal tissues. Cancer 1976;37(2 Suppl):1186-200.

10. Oanta A, Irimie M. Radiation recall dermatitis induced by tamoxifen. Bull Transilvania Univ Brasov VI Med Sci 2012;5:113-6.

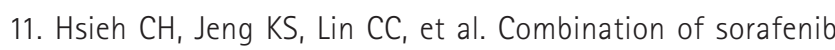
and intensity modulated radiotherapy for unresectable hepatocellular carcinoma. Clin Drug Investig 2009;29:65-71.

12. Hymes SR, Strom EA, Fife C. Radiation dermatitis: clinical presentation, pathophysiology, and treatment 2006. J Am Acad Dermatol 2006;54:28-46.

13. Yang $\mathrm{CH}_{\text {, Lin } W C}$, Chuang $\mathrm{CK}_{\text {, et }}$ al. Hand-foot skin reaction in patients treated with sorafenib: a clinicopathological study of cutaneous manifestations due to multitargeted kinase inhibitor therapy. Br J Dermatol 2008;158:592-6.

14. Ding $X$, Ji W, Li J, Zhang $X$, Wang L. Radiation recall pneumonitis induced by chemotherapy after thoracic radiotherapy for lung cancer. Radiat Oncol 2011;6:24.

15. Schweitzer VG, Juillard GJ, Bajada CL, Parker RG. Radiation recall dermatitis and pneumonitis in a patient treated with paclitaxel. Cancer 1995;76:1069-72.

16. Jeter MD, Janne PA, Brooks S, et al. Gemcitabine-induced radiation recall. Int J Radiat Oncol Biol Phys 2002;53:394-400.

17. Levy $A$, Hollebecque $A$, Bourgier $C$, et al. Targeted therapyinduced radiation recall. Eur J Cancer 2013;49:1662-8.

18. Ge J, Verma V, Hollander A, Langer C, Simone CB 2 nd. Pemetrexed-induced radiation recall dermatitis in a patient with lung adenocarcinoma: case report and literature review. J Thorac Dis 2016;8:E1589-E1593.

19. Ubukata M, Kamio T, Ohchi T, Noguchi E, Tsukada H, Kameoka S. Radiation recall dermatitis occurring 6 years and 4 months after breast-conserving surgery: a case report. Oncol Lett 2016;11:3071-4. 